\title{
Some Identities based on Success Runs of at Least Length $k$
}

\author{
Sonali Bhattacharya* \\ Symbiosis Centre for Management and Human Resource Development, (Deemed University), India
}

Submission: October 23, 2017; Published: January 17, 2018

*Corresponding author: Sonali Bhattacharya, Associate Professor, Symbiosis International (Deemed University), India, Tel: 91-20-22934304;

Email: sonali_bhattacharya@scmhrd.edu

\section{Abstract}

In this research paper, an attempt has been made to obtain alternative formulas for distributions of order $\mathrm{k}$ based on runs of at least length $k$. First by using binomial scheme and 'balls-into-cells' technique an alternative formula for distribution of binomial distribution of order $\mathrm{k}$ as defined by Goldstein [1]. Inverse Bionomial scheme was then used with 'ball-into-cells technique to obtain alternate distribution of Geometric distribution of order $\mathrm{k}$ and negative binomial distribution of order $\mathrm{k}$. The results were further extended to obtain Polya-Eggenberger distribution of order $\mathrm{k}$ and Inverse Polya-Eggenberger distributions of order $\mathrm{k}$. All results were verified for exactness of probability.

Keywords : Polya-Eggenberger sampling scheme; Distributions of order k; 'Balls-into-cells' technique

\section{Introduction}

Distribution of runs and successions in various situations have been under considerable studies due to their applications to reliability theory of consecutive systems Grifitth [2]; Papastravridis \& Sfkianakis [3], Sfkianakis, Kounias \& Hillaris [4], Papastravridis \& Koutras [5] \& Cai [6], start-up demonstration tests [7], molecular biology [1], theory of radar detection, time sharing systems and quality control [8-12]. There are different ways of computations and enumeration of number of runs:

1. Feller [13] defined ways of counting the runs of exactly length $\mathrm{k}$ as counting the number from scratch everytime a run occurs. For example the sequence $S S S|S F S S S| S S S \mid F$ contains 3 success runs of length 3

2. Goldstein [7] proposed the distribution of the number of success runs of at least length $\mathrm{k}$ until the $\mathrm{n}$-th trial. In this way of counting the number of runs of length 3 (or more), in the above example contains 2 success runs of length 3 (or more)

3. Schwager [14] and Ling $[15,16]$ studied the distributions on the number of overlapping runs of length $k$. In the enumeration scheme SSS $|S F S S S| S S S \mid F$ contains 6 overlapping success runs of length 3 .

4. Aki and Hirano [17] studied the distribution of success runs of exact length $k$. In the above example number of success runs of exact length 3 is 0 .

5. Philippou[18] obtained the distribution of the number of trials until the first occurrence of consecutive $k$ successes in Bernoulli trials with success probability as the geometric distribution of order $k,\left(G_{k}(x ; p)\right)$

In this paper, we have suggested alternative formulas for Binomial distribution of order $\mathrm{k}$ based on success runs of based on atleast length $k$ by using Balls-into-cells technique with direct sampling scheme with replacement. The same result was extended by using inverse sampling scheme to obtain alternative formula for Geometric distribution of order $k$ and negative Bionomial distribution of order $k$. Finally, using Polya Eggenberger sampling scheme we have obtained alternative formulas for Polya-Eggenberger distributions of order $k$ and Inverse Polya-Eggenberger distributions of order $k \quad[19,20]$.

Lemma: The number ways of distributing $r$ indistinguishable balls in $n$ cells such that each cell has atmost $(k-1)$ balls is given as:

$F^{(k)}(n ; r)=\sum_{i=0}^{\left\lceil\frac{r}{k}\right\rceil}(-1)^{i}\left(\begin{array}{l}n \\ i\end{array}\right)\left(\begin{array}{c}r+i k+n-1 \\ n-1\end{array}\right)$ (Riordan 1958) (1)

\section{Binomial distribution of order $\mathbf{k}$}

Let $X_{n}^{k}$ the number of success runs of length at least $k$ and $r$ be the number of success in n Bernoulli trials and $n-r$ be the number of failures.

Then,

Theorem 1:

$P\left(X_{n}^{k}=x\right)=\sum_{r=x k}^{a}\left(\begin{array}{c}n-r+x \\ x\end{array}\right) \sum_{j=0}^{\left[\frac{r-x k}{k}\right]}(-1)^{j}\left(\begin{array}{c}n-r+1 \\ j\end{array}\right)$ 
$\left(\begin{array}{c}n-x k-j k \\ n-r\end{array}\right) p^{r} q^{n-r}, \quad x=0,1, \ldots,\left[\frac{n}{k}\right]$

Where,

$a=\left[\frac{k x+(n+1)(k-1)}{k}\right]$

Proof: First consider $\mathrm{x}$ runs of exact length k successes each. $\mathrm{x}$ successes and $\mathrm{n}-\mathrm{r}$ failures can be arranged in $\left(\begin{array}{c}n-r+x \\ x\end{array}\right)$ ways. $r-x k$ remaining successes can be distributed into $n-r+1$ cells such that no cells have more than $k-1$ successes in

$F^{(k)}(n-r-1 ; r-x k)=\sum_{j=0}^{\left[\frac{r-x k}{k}\right]}(-1)^{j}\left(\begin{array}{c}n-r+1 \\ j\end{array}\right)\left(\begin{array}{c}n-x k-j k \\ n-r\end{array}\right)$

ways.

Thus, leading to the result. This is an alternative representation of probability distribution function of

For the maximum possible value of $r$,

Let us assume that there are $\mathrm{x}$ successes of exact length $k+(k-1)$ followed by a failure each.

Then the remaining number of cells formed by $n-r$ failures and $x$ successes will be $n-r+x+1$ each assumed to be having exactly $(k-1)$ successes.

So, $r \leq\left[\frac{x k+(n+1)(k-1)}{k}\right]$

\section{Some Examples:}

$$
\begin{aligned}
& \text { For } n=2, k=2, x=0,1 ; a=\left[\frac{3}{2}\right]=1 \\
& P\left(X_{2}^{2}=0\right)=\sum_{r=0}^{1} \sum_{j=0}^{\left[\frac{r}{2}\right]}(-1)^{j}\left(\begin{array}{c}
3-r \\
j
\end{array}\right)\left(\begin{array}{c}
2-2 j \\
2-r
\end{array}\right)\left(\begin{array}{c}
2-r \\
2-r
\end{array}\right) p^{r} q^{2-r} \\
& =q^{2}+2 p q \\
& P\left(X_{2}^{2}=0\right)=\sum_{r=2}^{2} \sum_{j=0}^{0}\left(\begin{array}{c}
2-2 \\
0
\end{array}\right)\left(\begin{array}{c}
2-2+1 \\
2-2
\end{array}\right) p^{2} \\
& =p^{2} \\
& P\left(X_{2}^{2}=0\right)+P\left(X_{2}^{2}=1\right)=(p+q)^{2}=1 \\
& \text { For } n=3, k=2, x=0,1 ; a=\left[\frac{4}{2}\right]=2 \\
& P\left(X_{3}^{2}=0\right)=\sum_{r=0}^{2} \sum_{j=0}^{\left[\frac{r}{2}\right]}(-1)^{j}\left(\begin{array}{c}
4-r \\
j
\end{array}\right)\left(\begin{array}{c}
3-2 j \\
3-r
\end{array}\right)\left(\begin{array}{c}
3-r \\
3-r
\end{array}\right) p^{r} q^{3-r} \\
& =q^{3}+3 p q^{2}+p^{2} q \\
& P\left(X_{3}^{2}=0\right)=\sum_{r=2}^{2} \sum_{j=0}^{\left[\frac{r-2}{2}\right]}(-1)^{j}\left(\begin{array}{c}
4-r \\
j
\end{array}\right)\left(\begin{array}{c}
1-2 j \\
3-r
\end{array}\right)\left(\begin{array}{c}
4-r \\
3-r
\end{array}\right) p^{r} q^{3-r} \\
& =2 p^{2} q+p^{3} \\
& \Rightarrow P\left(X_{3}^{2}=0\right)+P\left(X_{3}^{2}=1\right)=(q+p)^{3}=1 \\
& \text { For } n=10, k=2, x=0,1, \ldots, 5 \text {. } \\
& \text { For } x=0, a=\left[\frac{11}{2}\right]=5
\end{aligned}
$$

$$
\begin{aligned}
& P\left(X_{10}^{2}=0\right)=\sum_{r=0}^{5} \sum_{j=0}^{\left[\frac{r}{2}\right]}(-1)^{j}\left(\begin{array}{c}
11-r \\
j
\end{array}\right)\left(\begin{array}{c}
10-2 j \\
10-r
\end{array}\right)\left(\begin{array}{c}
10-r \\
10-r
\end{array}\right) p^{r} q^{10-r} \\
& =q^{10}+\left(\begin{array}{c}
10 \\
1
\end{array}\right) p q^{9}+\left(\begin{array}{c}
10 \\
2
\end{array}\right) p^{2} q^{8}-9 p^{2} q^{8}+\left(\begin{array}{c}
10 \\
3
\end{array}\right) p^{3} q^{7}-64 p^{3} q^{7} \\
& +\left(\begin{array}{c}
10 \\
4
\end{array}\right) p^{4} q^{6}-196 p^{4} q^{6}+21 p^{4} q^{6}+\left(\begin{array}{c}
10 \\
5
\end{array}\right) p^{5} q^{5}-246 p^{5} q^{5}
\end{aligned}
$$

For $x=1, a=6$

$$
\begin{aligned}
& P\left(X_{10}^{2}=1\right)=\sum_{r=2}^{6} \sum_{j=0}^{\left[\frac{r-2}{2}\right]}(-1)^{j}\left(\begin{array}{c}
11-r \\
j
\end{array}\right)\left(\begin{array}{c}
8-2 j \\
10-r
\end{array}\right)\left(\begin{array}{c}
11-r \\
10-r
\end{array}\right) p^{r} q^{10-r} \\
& =9 p^{2} q^{8}+64 p^{3} q^{7}+147 p^{4} q^{6}+120 p^{5} q^{5}+25 p^{6} q^{4}
\end{aligned}
$$

For $x=2, a=7$

$P\left(X_{10}^{2}=2\right)=\sum_{r=4}^{7} \sum_{j=0}^{\left[\frac{r-4}{2}\right]}(-1)^{j}\left(\begin{array}{c}11-r \\ j\end{array}\right)\left(\begin{array}{c}6-2 j \\ 10-r\end{array}\right)\left(\begin{array}{c}12-r \\ 10-r\end{array}\right) p^{r} q^{10-r}$

$=28 p^{4} q^{6}+126 p^{5} q^{5}+150 p^{6} q^{4}+40 p^{7} q^{3}$

For $x=3, a=8$

$P\left(X_{10}^{2}=3\right)=\sum_{r=6}^{8} \sum_{j=0}^{\left.\frac{r-6}{2}\right]}(-1)^{j}\left(\begin{array}{c}11-r \\ j\end{array}\right)\left(\begin{array}{c}4-2 j \\ 10-r\end{array}\right)\left(\begin{array}{c}13-r \\ 10-r\end{array}\right) p^{r} q^{10-r}$

$=35 p^{6} q^{4}+80 p^{7} q^{3}+30 p^{8} q^{2}$

For $x=4, a=9$

$$
\begin{aligned}
& P\left(X_{10}^{2}=4\right)=\sum_{r=6}^{9} \sum_{j=0}^{\left[\frac{r-8}{2}\right]}(-1)^{j}\left(\begin{array}{c}
11-r \\
j
\end{array}\right)\left(\begin{array}{c}
2-2 j \\
10-r
\end{array}\right)\left(\begin{array}{c}
14-r \\
10-r
\end{array}\right) p^{r} q^{10-r} \\
& =15 p^{8} q^{2}+10 p^{9} q
\end{aligned}
$$

For $x=5, a=10$

$$
\begin{aligned}
& P\left(X_{10}^{2}=5\right)=\sum_{r=10}^{10} \sum_{j=10}^{0}(-1)^{0}\left(\begin{array}{l}
0 \\
0
\end{array}\right)\left(\begin{array}{l}
5 \\
0
\end{array}\right) p^{10}=p^{10} \\
& P\left(X_{10}^{2}=0\right)+P\left(X_{10}^{2}=1\right)+P\left(X_{10}^{2}=2\right)+P\left(X_{10}^{2}=3\right)+P\left(X_{10}^{2}=4\right)+P\left(X_{10}^{2}=5\right) \\
& =(q+p)^{10}=1
\end{aligned}
$$

\section{Geometric distribution of order $\mathbf{k}$}

Let the first success run of length k occurs at $N_{k}^{\text {th }}$ trial. Then,

\section{Theorem 2}

$P\left(X_{10}^{2}=3\right)=\sum_{r=k}^{a} \sum_{j=0}^{\left[\frac{r-k}{k}\right]}(-1)^{j}\left(\begin{array}{c}n-r \\ j\end{array}\right)\left(\begin{array}{c}n-k-j k-1 \\ n-r-1\end{array}\right) p^{r} q^{n-r}$

$x \geq k$ (3)

Where, $a=\left[\frac{k+n(k-1)}{k}\right]$

Proof: Let there be $r$ successes in

$\underbrace{\ldots \ldots \ldots .}_{n-k-1} F \underbrace{S S \ldots S}_{k}$ 
The number of ways of distributing $r-k$ successes preceding failures $n-r$ such that not more than $k-1$ successes occur proceeding the $n-r$ failures then by using (1) and replacing $n$ by $n-r$ and $r$ by $r-k$ is given by

$$
F^{(k)}(n-r ; r-k)=\sum_{j=0}^{\left[\frac{r-k}{k}\right]}(-1)^{j}\left(\begin{array}{c}
n-r \\
j
\end{array}\right)\left(\begin{array}{c}
n-k-j k-1 \\
n-r-1
\end{array}\right) .
$$

Hence, the result. This is thus an alternative formula for Geometric distribution of order $k$ given by Philippou [18].

Remark 1: For $k=1$ (3) gives the probability mass function of Geometric Distribution.

$$
\begin{aligned}
& \text { An Example } \\
& \qquad \begin{array}{l}
\text { For } n=10, k=3, a=\left[\frac{3+20}{3}\right]=\left[\frac{23}{3}\right]=7 \\
P\left(N_{3}=10\right)=\sum_{r=3}^{7} \sum_{j=0}^{\left[\frac{r-3}{3}\right]}(-1)^{j}\left(\begin{array}{c}
10-r \\
j
\end{array}\right)\left(\begin{array}{c}
6-3 j \\
9-r
\end{array}\right) p^{r} q^{10-r} \\
=p^{3} q^{7}+6 p^{4} q^{6}+15 p^{5} q^{5}+16 p^{6} q^{4}+6 p^{7} q^{3}
\end{array}
\end{aligned}
$$

\section{Negative Binomial Distribution of order $k$}

Let the $x^{\text {th }}$ run of length k occurs at the $N_{k}^{x}-t h$ trial i.e.,

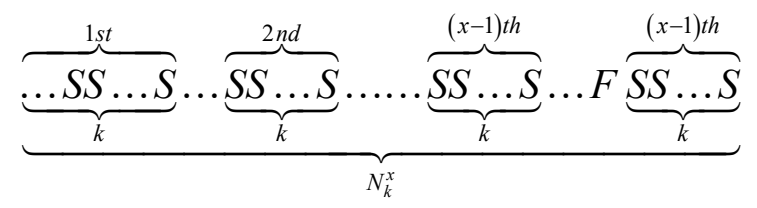

Theorem 3

$$
P\left(N_{k}^{x}=n\right)=\sum_{r=x k}^{a} \sum_{j=0}^{\left[\frac{r-x k}{k}\right]}(-1)^{j}\left(\begin{array}{c}
n-r \\
j
\end{array}\right)\left(\begin{array}{c}
n-x k-j k-1 \\
n-r-1
\end{array}\right)\left(\begin{array}{c}
n-r+x-1 \\
x-1
\end{array}\right) p^{r} q^{n-r}
$$

$x \geq n k \quad(3)$

\section{Proof}

Where, $a=\left[\frac{x k+n(k-1)}{k}\right]$

Let there be $r$ successes and $n-r$ failures. $r-x k$ successes are to be distributed into $n-r$ cells preceding the $n-r$ failures such that no cell receives more than $k-1$ successes which is given by (1) replacing $r$ by $r-x k$ and $n$ by as

$$
F^{(k)}(n-r ; r-x k)=\sum_{j=0}^{\left[\frac{r-x k}{k}\right]}(-1)^{j}\left(\begin{array}{c}
n-r \\
j
\end{array}\right)\left(\begin{array}{c}
n-x k-j k-1 \\
n-r-1
\end{array}\right)
$$

Hence, the probability. Further, $x k+(n-r)(k-1) \geq r$. Hence, $r \leq\left[\frac{x k+n(k-1)}{k}\right]=a$

This is thus an alternative formula for Negative Binomial distribution of order given by Philippou [18].

\section{Some examples}

For $k=1 \rightarrow a=x$ and $r=x$

$$
P\left(N_{1}^{x}=n\right)=\left(\begin{array}{l}
n-x-1 \\
n-x-1
\end{array}\right)\left(\begin{array}{l}
n-1 \\
x-1
\end{array}\right) p^{x} q^{n-x} \text { is the probability mass }
$$
function of Negative Binomial Distribution

$$
\begin{aligned}
& \text { For } n=8, k=2, x=2, a=\left[\frac{4+8}{2}\right]=6 \\
& P\left(N_{2}^{2}=8\right)=\sum_{r=4}^{6} \sum_{j=0}^{\left.\frac{r-4}{2}\right]}(-1)^{j}\left(\begin{array}{c}
8-r \\
j
\end{array}\right)\left(\begin{array}{c}
3-2 j \\
7-r
\end{array}\right)\left(\begin{array}{c}
9-r \\
1
\end{array}\right) p^{r} q^{n-r} \\
& =5 p^{4} q^{4}+12 p^{5} q^{3}+3 p^{6} q^{2}
\end{aligned}
$$

\section{Polya-Eggenberger Distribution of order K}

Let us assume an urn contains $a$ white and $b$ black balls. A ball is drawn, its colour is noted and it is returned to the urn with $S$ additional balls of the same colour. The process is continued till $n$ balls have been drawn.

Let $X_{n ; k}^{s}$ be the number of white ball runs of at least length $k$ in $n$ trials. Then,

Theorem 4: $P\left(X_{n ; k}^{s}=x\right)=\sum_{r=x k}^{a}\left(\begin{array}{c}n-r+x \\ x\end{array}\right) \sum_{j=0}^{\left[\frac{r-4 x k}{k}\right]}(-1)^{j}\left(\begin{array}{c}n-r+1 \\ j\end{array}\right)$

$\left(\begin{array}{c}n-x k-j k \\ n-r\end{array}\right) \frac{a^{(r, s)} b^{(n-r, s)}}{(a+b)^{(n, s)}}$

Where,

$$
a=\left[\frac{k x+(n+1)(k-1)}{k}\right]
$$

The proof follows from theorem 1 . This is thus an alternative formula for Polya distribution of order given by Charalambides [18] Sen et al. [19].

\section{Some examples}

For $n=7, k=2, x=0,1,2,3$.

$P\left(X_{7 ; 2}^{s}=x\right)=\sum_{r=2 x}^{a}\left(\begin{array}{c}7-r+x \\ x\end{array}\right) \sum_{j=0}^{\left[\frac{r-2 x}{2}\right]}(-1)^{j}\left(\begin{array}{c}8-r \\ j\end{array}\right)\left(\begin{array}{c}7-2 x-2 j \\ 7-r\end{array}\right) \frac{a^{(r, s)} b^{(7-r, s)}}{(a+b)^{(7, s)}}$ $a=x+4$

$P\left(X_{7 ; 2}^{s}=x\right)=\sum_{r=0}^{4}(-1)^{j}\left(\begin{array}{c}8-r \\ j\end{array}\right)\left(\begin{array}{c}7-2 x-2 j \\ 7-r\end{array}\right) \frac{a^{(r, s)} b^{(7-r, s)}}{(a+b)^{(7, s)}}$ $=\frac{b^{(7, s)}}{(a+b)^{(7, s)}}+7 \frac{a b^{(6, s)}}{(a+b)^{(7, s)}}+15 \frac{a^{(2, s)} b^{(5, s)}}{(a+b)^{(7, s)}}+10 \frac{a^{(3, s)} b^{(4, s)}}{(a+b)^{(7, s)}}+\frac{a^{(4, s)} b^{(3, s)}}{(a+b)^{(7, s)}}$ $P\left(X_{7 ; 2}^{s}=1\right)=\sum_{r=2}^{5}\left(\begin{array}{c}8-r \\ 1\end{array}\right) \sum_{j=0}^{\left.\frac{r-2 x}{2}\right]}(-1)^{j}\left(\begin{array}{c}8-r \\ j\end{array}\right)\left(\begin{array}{c}5-2 j \\ 7-r\end{array}\right) \frac{a^{(r, s)} b^{(7-r, s)}}{(a+b)^{(7, s)}}$ $=6 \frac{a^{(2, s)} b^{(5, s)}}{(a+b)^{(7, s)}}+25 \frac{a^{(3, s)} b^{(4, s)}}{(a+b)^{(7, s)}}+24 \frac{a^{(4, s)} b^{(3, s)}}{(a+b)^{(7, s)}}+3 \frac{a^{(5, s)} b^{(2, s)}}{(a+b)^{(7, s)}}$ $P\left(X_{7 ; 2}^{s}=2\right)=\sum_{r=4}^{6}\left(\begin{array}{c}9-r \\ 2\end{array}\right) \sum_{j=0}^{\left[\frac{r-4}{2}\right]}(-1)^{j}\left(\begin{array}{c}8-r \\ j\end{array}\right)\left(\begin{array}{c}3-2 j \\ 7-r\end{array}\right) \frac{a^{(r, s)} b^{(7-r, s)}}{(a+b)^{(7, s)}}$ $=10 \frac{a^{(4, s)} b^{(3, s)}}{(a+b)^{(7, s)}}+18 \frac{a^{(5, s)} b^{(2, s)}}{(a+b)^{(7, s)}}+3 \frac{a^{(6, s)} b^{(1, s)}}{(a+b)^{(7, s)}}$ 
$P\left(X_{7 ; 2}^{s}=3\right)=\sum_{r=6}^{7}\left(\begin{array}{c}10-r \\ 3\end{array}\right) \sum_{j=0}^{\left[\frac{r-6}{2}\right]}(-1)^{j}\left(\begin{array}{c}8-r \\ j\end{array}\right)\left(\begin{array}{c}1-2 j \\ 7-r\end{array}\right) \frac{a^{(r, s)} b^{(7-r, s)}}{(a+b)^{(7, s)}}$

$=4 \frac{a^{(6, s)} b^{(1, s)}}{(a+b)^{(7, s)}}+\frac{a^{(7, s)}}{(a+b)^{(7, s)}}$

$\stackrel{\text { yields }}{\longrightarrow} P\left(X_{7 ; 2}^{s}=0\right)+P\left(X_{7 ; 2}^{s}=1\right)+P\left(X_{7 ; 2}^{s}=2\right)+P\left(X_{7 ; 2}^{s}=x\right)$

$=\frac{b^{(7, s)}}{(a+b)^{(7, s)}}+7 \frac{a b^{(6, s)}}{(a+b)^{(7, s)}}+21 \frac{a^{(2, s)} b^{(5, s)}}{(a+b)^{(7, s)}}+35 \frac{a^{(3, s)} b^{(4, s)}}{(a+b)^{(7, s)}}+35 \frac{a^{(4, s)} b^{(3, s)}}{(a+b)^{(7, s)}}$

$+21 \frac{a^{(5, s)} b^{(2, s)}}{(a+b)^{(7, s)}}+7 \frac{a^{(6, s)} b^{(1, s)}}{(a+b)^{(7, s)}}+\frac{a^{(7, s)}}{(a+b)^{(7, s)}}=\sum_{x=0}^{7}\left(\begin{array}{l}7 \\ x\end{array}\right) \frac{a^{(x, s)} b^{(7-x, s)}}{(a+b)^{(7, s)}}=1$

\section{Inverse polya-eggenberger distribution of order $\mathbf{k}$}

Let us assume an urn contains $a$ white and $b$ black balls. Balls are drawn one-by-one with $S$ replacement along with additional balls of the same colour of ball drawn. The process is continued till $n$ balls have been drawn [21].

Let $N_{k}^{x, s}$ be the number of trials required for $x^{\text {th }}$ run of $k$ white balls to occur $\left(N_{k}^{x, 0}=N_{k}^{x}\right)$. Then,

Then, using theorem 3 we have,

\section{Theorem 5}

$\left(N_{k}^{x, s}=n\right)=\sum_{r=k}^{a} \sum_{j=0}^{\left[\frac{r-x k}{k}\right]}(-1)^{j}\left(\begin{array}{c}n-r \\ j\end{array}\right)\left(\begin{array}{c}n-k-j k-1 \\ n-r-1\end{array}\right)\left(\begin{array}{c}n-r+x-1 \\ x-1\end{array}\right) \frac{a^{(r, s)} b^{(n-r, s)}}{(a+b)^{(n, s)}}$

$x \geq n k \quad(6)$

Where, $a=\left[\frac{x k+n(k-1)}{k}\right]$ This is thus an alternative formula for Inverse Polya distribution of order $k$ given by

\section{Some examples}

For $n=9, k=3, x=2$

$\left(N_{3}^{2, s}=9\right)=\sum_{r=6}^{8} \sum_{j=0}^{\left[\frac{r-6}{3}\right]}(-1)^{j}\left(\begin{array}{c}9-r \\ j\end{array}\right)\left(\begin{array}{c}2-3 j \\ 8-r\end{array}\right)\left(\begin{array}{c}10-r \\ x-1\end{array}\right) \frac{a^{(r, s)} b^{(n-r, s)}}{(a+b)^{(n, s)}}$

$=4 \frac{a^{(6, s)} b^{(3, s)}}{(a+b)^{(9, s)}}+6 \frac{a^{(7, s)} b^{(2, s)}}{(a+b)^{(9, s)}}+2 \frac{a^{(8, s)} b^{(1, s)}}{(a+b)^{(9, s)}}$ which include following arrangements of white and black balls:

\section{$W W B W W W W W W, W W W W W B W W W$,}

two arrangements of 1 black \& 8 white balls

BWBWWWWW, $W B B W W W W W W, W B W W W B W W W, W W W W B B W W W, B W W W W B W W W, W W W B W B W W W$

, $B B B W W W W W W, B B W W W B W W W, W W W B B B W W W, B W W W B B W W W$

four arrangements of 3 black \& 6 white balls

For $n=11, k=2, x=3$

$\left(N_{3}^{2, s}=11\right)=\sum_{r=6}^{8} \sum_{j=0}^{\left[\frac{r-6}{3}\right]}(-1)^{j}\left(\begin{array}{c}11-r \\ j\end{array}\right)\left(\begin{array}{c}4-2 j \\ 10-r\end{array}\right)\left(\begin{array}{c}13-r \\ 2\end{array}\right) \frac{a^{(r, s)} b^{(n-r, s)}}{(a+b)^{(n, s)}}$

$=21 \frac{a^{(6, s)} b^{(5, s)}}{(a+b)^{(11, s)}}+60 \frac{a^{(7, s)} b^{(4, s)}}{(a+b)^{(11, s)}}+30 \frac{a^{(8, s)} b^{(3, s)}}{(a+b)^{(11, s)}}$

\section{References}

1. Goldstein L (1990) Poisson approximation and DNA sequence matching. Communications in Statistics-Theory and Methods 19(11): 4167-4179.

2. Griffith WS (1986) On consecutive k-out-of-n failure systems and their generalizations. Reliability and quality control 20: 157-165.

3. Papastavridis SG, Sfakianakis ME (1991) Optimal-arrangement and importance of the components in a consecutive-k-out-of-r-from-n: F system. IEEE Transactions on Reliability 40(3): 277-279.

4. Sfakianakis M, Kounias S, Hillaris A (1992) Reliability of a consecutive k-out-of-r-from-n: F system. IEEE Transactions on Reliability 41(3): 442-447.

5. Papastavridis SG, Koutras MV (1993) Bounds for reliability of consecutive k-within-m-out-of-n: F systems. Reliability. IEEE Transactions 42(1): 156-160.

6. Cai J (1994) Reliability of a large consecutive-k-out-of-r-from-n: F system with unequal component-reliability. IEEE transactions on reliability 43(1): 107-111.

7. Hahn GJ, GAGE JB (1983) Evaluation of a start-up demonstration test. Journal of Quality Technology 15: 103-106.

8. Greenberg I (1970) The first occurrence of $\mathrm{n}$ successes in $\mathrm{N}$ trials. Technometrics 12(3): 627-634.

9. Saperstein B (1973) On the occurrence of $n$ successes within $N$ Bernoulli trials. Technometrics 15(4): 809-818.

10. Nelson JB (1978) Minimal-order models for false-alarm calculations on sliding windows. Aerospace and Electronic Systems. IEEE Transactions (2): 351-363.

11. Mirstik AV (1978) Multistatic radar binomial detection. IEEE Transactions on Aerosp Electron Syst 14(1): 103-108.

12. Glaz J (1983) Moving window detection for discrete data. IEEE Transactions on Information Theory. 29(3): 457-462.

13. Feller W (1968) An Introduction to Probability Theory .

14. Schwager SJ (1983) Run probabilities in sequences of Markovdependent trials. Journal of the American Statistical Association 78(381): 168-175.

15. Ling KD (1988) On binomial distributions of order k. Statistics \& probability letters 6(4): 247-250.

16. Ling KD (1989) A new class of negative binomial distributions of order k. Statistics \& Probability Letters 7(5): 371-376.

17. Aki S, Hirano K (1996) Lifetime distribution and estimation problems of consecutive-k-out-of-n: F systems. Annals of the Institute of Statistical Mathematics 48(1): 185-199.

18. Philippou AN (1983) The Poisson and compound Poisson distributions of order $\mathrm{k}$ and some of their properties. Zapiski Nauchnykh Seminarov POMI, 130:175-180.

19. Charalambides CA (1986) On discrete distributions of orderk. Annals of the Institute of Statistical Mathematics 38(1): 557-568.

20. Sen K, Lata AM, Chakraborty S (2002) Generalized Polya-Eggenberger model of order $\mathrm{k}$ via lattice path approach. Journal of statistical planning and inference 102(2): 467-476.

21. Riordan J (1978) An introduction to combinatorial analysis. Princeton. 

CC) $\begin{aligned} & \text { This work is licensed under Creative } \\ & \text { Commons Attribution } 4.0 \text { Licens } \\ & \text { DOI: } 10.19080 / \text { BBOAJ.2018.04.555634 }\end{aligned}$

\section{Your next submission with Juniper Publishers} will reach you the below assets

- Quality Editorial service

- Swift Peer Review

- Reprints availability

- E-prints Service

- Manuscript Podcast for convenient understanding

- Global attainment for your research

- Manuscript accessibility in different formats

( Pdf, E-pub, Full Text, Audio)

- Unceasing customer service

Track the below URL for one-step submission https://juniperpublishers.com/online-submission.php 